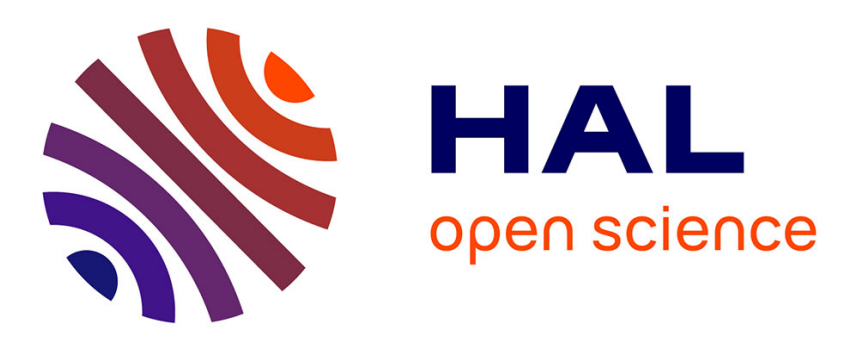

\title{
Direct observation of subpicosecond vibrational dynamics in photoexcited myoglobin
}

C. Ferrante, E. Pontecorvo, Giulio Cerullo, Marten H. Vos, T. Scopigno

\section{To cite this version:}

C. Ferrante, E. Pontecorvo, Giulio Cerullo, Marten H. Vos, T. Scopigno. Direct observation of subpicosecond vibrational dynamics in photoexcited myoglobin. Nature Chemistry, 2016, 8, pp.1137-1143 10.1038/nchem.2569 . hal-01612918

HAL Id: hal-01612918

https://hal-polytechnique.archives-ouvertes.fr/hal-01612918

Submitted on 30 Nov 2017

HAL is a multi-disciplinary open access archive for the deposit and dissemination of scientific research documents, whether they are published or not. The documents may come from teaching and research institutions in France or abroad, or from public or private research centers.
L'archive ouverte pluridisciplinaire HAL, est destinée au dépôt et à la diffusion de documents scientifiques de niveau recherche, publiés ou non, émanant des établissements d'enseignement et de recherche français ou étrangers, des laboratoires publics ou privés. 


\title{
Direct observation of sub-picosecond vibrational dynamics in photoexcited myoglobin
}

\author{
C. Ferrante ${ }^{1}$, E. Pontecorvo ${ }^{1}$, G. Cerullo ${ }^{2}$, M. H. Vos ${ }^{3}$, T. Scopigno ${ }^{1}$ \\ ${ }^{1}$ Dipartimento di Fisica, Università di Roma "La Sapienza", I-00185, Roma, Italy \\ ${ }^{2}$ IFN-CNR, Dipartimento di Fisica, Politecnico di Milano, \\ P.zza L. da Vinci 32, 20133 Milano, Italy and \\ ${ }^{3}$ LOB, Ecole Polytechnique, CNRS, INSERM, Université Paris-Saclay, 91128 Palaiseau Cedex, France
}

\begin{abstract}
Determining the initial pathway for ultrafast energy redistribution within biomolecules is a challenge. Heme proteins, where energy can be locally deposited in the heme moiety using short light pulses, are convenient model systems to address this issue. Due to lack of experiments combining structural sensitivity and time resolution, however, alternative hypotheses coexist concerning the interplay between energy flow among highly excited vibrational levels and potential concomitant electronic processes. By developing a Femtosecond Stimulated Raman setup, endowed with the necessary tunability to take advantage of different resonance conditions, here we visualize the temporal evolution of energy redistribution over different vibrational modes in myoglobin. We establish that vibrational energy initially stored in the highly excited Franck-Condon manifold is transferred with different timescales into low and high frequency modes, prior to slow dissipation through the protein. These findings demonstrate that a newly proposed mechanism involving population dynamics of specific vibrational modes settles the controversy on the existence of transient electronic intermediates.
\end{abstract}

Unraveling energy flow and redistribution pathways within biological macromolecules is essential for understanding the physicochemical regime in which biological reactions occur ${ }^{1-4}$. Photochemical methods are in principle ideally suited to study such processes, because ultrashort light pulses can be used to deposit excess energy in a controlled, localized way on a time scale faster than vibrational diffusion ${ }^{5}$. Heme proteins exert a wide and still expanding range of biological functions, including catalysis, ligand transport and sensing, and especially their prototypical representative myoglobin $(\mathrm{Mb})$ has become a model system for protein dynamics ${ }^{6}$. Heme photoexcitation (sketched in Fig. 1a) can lead to the rupture of the heme-ligand bond followed by structural changes in the heme, the protein moiety and the ligand position, spanning over several orders of magnitude in time ${ }^{3,6}$. The corresponding energy relaxation processes have been extensively studied with a variety of ultrafast techniques, such as time-resolved infrared ${ }^{7}$ and visible ${ }^{8-10}$ absorption, as well as by Impulsive Raman ${ }^{11}$ and Coherent Emission Interferometry ${ }^{12}$. Using Time-Resolved Resonance Raman $\left(\mathrm{TR}^{3}\right)$ and Transient Raman Resonance Spectroscopy (TRRS), heme cooling has been observed on the timescales of a few picoseconds by looking at either vibrational shift ${ }^{13-15}$ or the Stokes/Antistokes ratio ${ }^{2,16,17}$ of the totally symmetric in-plane breathing modes with large cross section $\left(\nu_{4} \text { and } \nu_{7}\right)^{18}$. The precise mechanism ruling vibrational energy redistribution following photoactivation, however, is obscured by several concomitant processes on the subpicosecond timescale: lysis of the chemical bond between the heme and external ligands ${ }^{19,20}$, excited state dynamics and conformational changes $^{21}$. In particular, owing to the difficulties to experimentally achieve simultaneous structural sensitivity and time resolution, contrasting hypotheses (schematically depicted in Fig. 1b) have been put forward for the dynamics at 50 fs $<t<5$ ps with respect to the possible contributions of intermediate heme electronic states $^{7,8,10,14,17,19,21-25}$.

To be able to address these issues we developed a novel Femtosecond Stimulated Resonance Raman setup (FSRRS) that uniquely combines high temporal precision and frequency resolution with fine tunability all over the heme proteins absorption spectrum. This approach allows us to derive the following scenario for ultrafast energy redistribution in myoglobin: following light absorption, energy is promptly transferred into a manifold of highly excited vibrational levels of the electronic ground state. From this manifold, vibrationally excited levels of low-frequency modes are populated quasiinstantaneously, and are thermally equilibrated within $\sim 300$ fs. In striking contrast, the higher excited levels of high-frequency modes are populated in a few hundred femtoseconds, and relax in several picoseconds, constituting a bottleneck for overall equilibration of the heme prior to energy dissipation into the protein moiety. A model is developed that quantitatively accounts for the stimulated Raman as well as for the spectrally resolved transient visible absorption data, and that does not require intermediate electronic states of the heme.

\section{Results}

The two main absorption bands of Myoglobin, the Qband ( $\approx 560 \mathrm{~nm}$ ) corresponding to the low-energy transition and the higher-lying Soret band $(\approx 430 \mathrm{~nm})$, are shown in Fig. 2d, for both the deoxy and CO-bound forms. We preliminarily performed a Transient Absorption (TA) experiment in deoxy $\mathrm{Mb}$, pumped in the Qband and probed in a broad region across the Soret band, reported in Fig. 2. A broad photoinduced absorption 

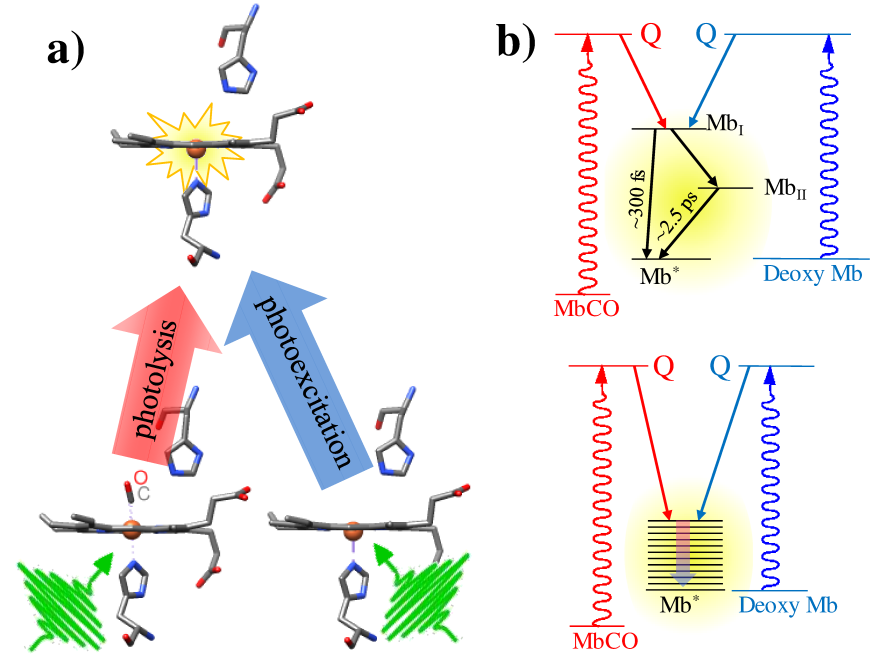

FIG. 1: Heme photolysis/photoexcitation.

a)

Sketch of heme pocket during photolysis/photoexcitation of $\mathrm{MbCO} /$ deoxy $\mathrm{Mb}$, respectively. b) Two alternative relaxation pathways following $\mathrm{Q}$ band absorption of the pump photon by the reactant: the first is based on the existence of transient electronic states $\mathrm{Mb}_{I}$ and $\mathrm{Mb}_{I I}$ on the way back to the ground state, the second entirely develops as direct deexcitation within a hot electronic ground state.

band develops to the red of the bleached Soret band $(S$, $\approx 435 \mathrm{~nm}$ ), with a maximum at $\approx 450 \mathrm{~nm}$ (Fig. $2 \mathrm{~b}$ ), and decays in $\approx 3$ ps. A further-redshifted tail disappears on a much faster timescale, $\approx 300 \mathrm{fs}$ (Fig. $2 \mathrm{c}$ ); as extensively discussed in the literature, these TA features are compatible with either the existence of intermediate electronic species ${ }^{7,14,21,23,24}$ or with a hot ground state vibrational relaxation pathway ${ }^{8,10}$, as illustrated in Fig. 1b. Solving this long standing controversy requires, on a more general ground, unravelling the redistribution of energy deposited locally in a prototypical cofactor the heme of myoglobin- prior to the flow of heat into the protein moiety. To this purpose, we resorted to transient Raman spectroscopy. As classical $\mathrm{TR}^{3}$ spectroscopy cannot provide subpicosecond time resolution $(\Delta t)$ if an acceptable frequency resolution $(\Delta \omega)$ has to be maintained ( $\hbar \Delta \omega \Delta t \geq 15 \mathrm{~cm}^{-1} \mathrm{ps}$, due to the transform limit restriction holding for the time duration and bandwidth of a single light pulse), we developed a dedicated FSRRS setup with wavelength-tunable Raman pulses in the challenging blue spectral region. The basic concept of femtosecond stimulated Raman scattering ${ }^{26}$ is to use a femtosecond actinic pump pulse to initiate photochemistry of interest (centered at $560 \mathrm{~nm}$ in our case), and a picosecond Raman pulse (RP) jointly with a femtosecond broadband probe (Stokes pulse) to stimulate broadband

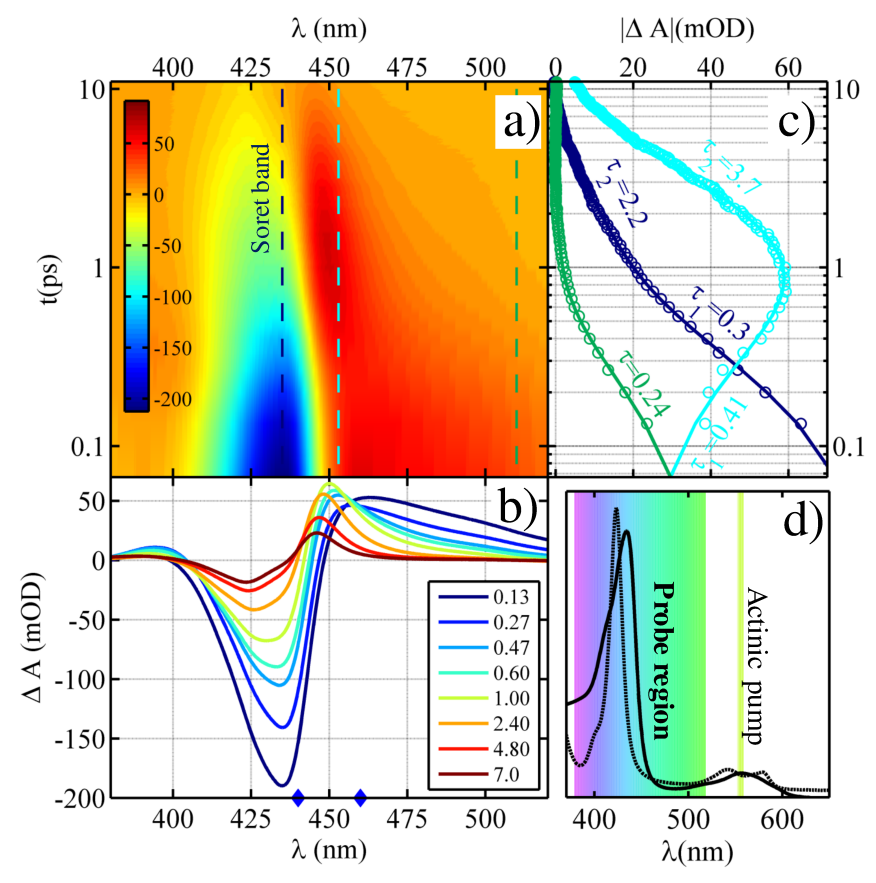

FIG. 2: Transient absorption experiment in deoxy myoglobin. a) Transient absorption map, as a function of the probe wavelength and delay, of deoxy $\mathrm{Mb}$ in the Soret region, upon $\mathrm{Q}$ band photoexcitation. The color scale is in mOD. b) transient absorption spectra for selected time delays (indicated in ps in the legend). Raman pulse wavelengths used for the FSRRS experiment are also shown as blue diamonds. c) selected kinetics for the three representative probe wavelengths $(435,453,510 \mathrm{~nm})$ indicated as dashed lines of corresponding color in panel a. The reported timescales are obtained by exponential best fits. d) Absorption spectra of CO-bound and deoxy Mb. Pump and probe wavelength regions are also indicated.

coherent Raman signals in a wide vibrational range (100 2000) $\mathrm{cm}^{-1}$. The key advantage of the technique is that, although the generated coherence is read out averaged over the vibrational dephasing time, by controlling the gating of the Raman coherence the dephasing time window can be moved with uncompromised time precision $(50 \mathrm{fs})$. As a result, tracking ultrafast structural dynamics becomes possible under the constraint imposed by the Heisenberg principle ${ }^{27-29}$.

FSRRS maps for both carbonmonoxy myoglobin $(\mathrm{MbCO})$ and deoxy-Mb are shown in Fig. 3. As a first general remarkable observation, our data clearly show only red shifts of the deoxy-ground state bands (quantified in Supplementary Fig. 2), highly suggestive of anharmonicity, and neither additional modes nor disappearance of ground state vibrations. This implies that the TA features of Fig. 2 reflect vibrationally excited levels of the ground state rather than population of transient electronic states. In the following we will demonstrate that the ensemble of observations can be understood in a consistent picture. 


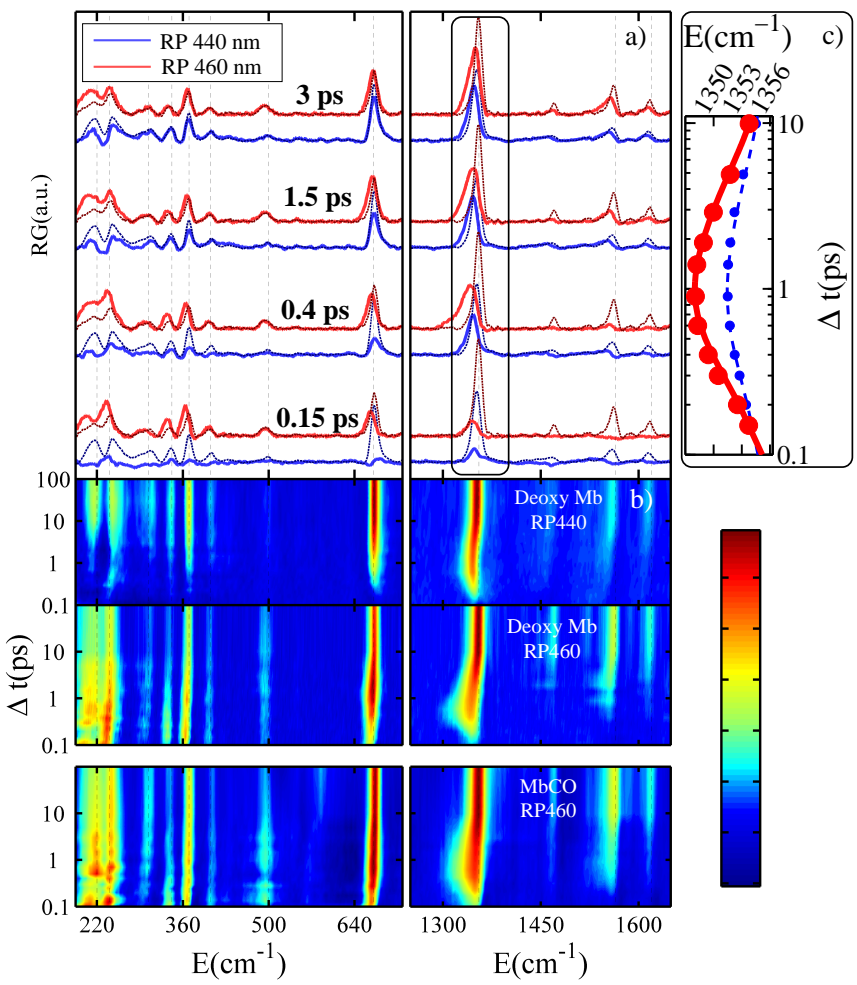

FIG. 3: FSRRS snapshots of vibrational energy flow in Mb. a) FSRRS spectra of deoxy-Mb (solid lines) after subtraction of the non-excited molecular fraction and corrected for probe chirp are shown for different time delays with the $\mathrm{RP}$ at $460 \mathrm{~nm}$ (red) and at $440 \mathrm{~nm}$ (blue). Reference spectra in absence of the actinic pulse are also reported (dashed lines). b) Corresponding Raman colormaps, the $\mathrm{MbCO}$ with $\mathrm{RP}$ at $460 \mathrm{~nm}$ is also shown. The vertical dashed lines are guides to the eyes. The data demonstrate slower population and decay of the vibrational excited levels of the high-frequency modes, compared to the low-frequency modes. c) kinetics of the $\nu_{4}$ energy for deoxy-Mb (red circles), well described by a biexponential fit with time constants of 0.3 and 6 ps (red line). Data for $\mathrm{MbCO}$ are also shown for comparison (blue).

When the RP is centered at $460 \mathrm{~nm}$, an instantaneous photoinduced red shift of the low frequency modes $(\leq 700$ $\mathrm{cm}^{-1}$, cf. $\nu_{8}$ in Fig. 4b) is observed followed by a recovery of the ground state frequencies in $\approx 300 \mathrm{fs}$. This recovery occurs concomitant with a decay of the amplitude of the Raman bands. By contrast, upon tuning the $\mathrm{RP}$ at $440 \mathrm{~nm}$, the intensity buildup of the ground state low frequency modes is retarded by a few hundred fs.

For the high frequency modes $\left(1000-1600 \mathrm{~cm}^{-1}\right.$, cf. $\nu_{4}$ in Fig. 4a) a different pattern is observed. These modes build up within the first 300 fs for both RP tuning conditions. In addition, the transient red-shift is not instantaneous, it rather develops within the first ps, as clearly observed with the $\approx 460 \mathrm{~nm} \mathrm{RP}$ and, to a lesser extent, with the $\approx 440 \mathrm{~nm}$ RP. On the timescale of a few picosec- onds the frequencies shift back to the cold ground state values (Fig. 3, inset), in agreement with $\mathrm{TR}^{3}$ experiments on the $\nu_{4}$ mode $^{23}$ that were interpreted in terms of vibrational cooling, related to anharmonic mode coupling with low frequency modes ${ }^{14}\left(\approx 500 \mathrm{~cm}^{-1}\right)$. Our observation rationalizes previous TRRS experiments ${ }^{17}$, specifically the peculiar shape of the $\nu_{4}$ vibration (see Supplementary Discussion for full details). The improved time-energy resolution of our FSRRS experiment enables a detailed inspection of the dominant $\nu_{4}$ band $(\approx 1355$ $\mathrm{cm}^{-1}$ in deoxy-Mb), which exhibits a red shift due to the presence of a distinct transient satellite to the red side of the ground state vibrational frequency. Remarkably the relative weight is markedly enhanced for the $\mathrm{RP}$ at $460 \mathrm{~nm}$ (Fig. 5b). The effect is present in both $\mathrm{MbCO}$ and deoxy-Mb, albeit with different amplitudes (Fig. 3) due to the fact that in the former case part of the photoexcitation energy quantum is spent to dissociate the ligand, resulting in a reduced heating. This establishes the common nature of energy redistribution in $\mathrm{MbCO}$ and deoxy-Mb, and of their nearly coincident spectra even at the shortest probed time delay. Hence, we focus here on the latter case, particularly illustrative due to the coincidence of the initial and final states.

\section{Discussion}

Detailed information on the ultrafast energy flow within the heme can be obtained from the dependence of the FSRRS spectra on the RP tuning. In general, transitions starting from vibrationally excited states $n>0$ are enhanced by the increase of the Boltzmann population factor. In the presence of anharmonicity, the vibrational energy levels are unevenly spaced. This gives rise to distinct vibrational spectral features associated with hot photoproducts, i.e., hot bands, that are red-shifted with respect to the fundamental transition starting from $n=0^{30}$. Accordingly, if the energy of the RP is tuned below the energy difference between the vibrational ground state $\left(|0\rangle_{g}\right)$ and the Soret band, the cross section associated with the transition from a vibrationally excited state (i.e. $|1\rangle_{g} \rightarrow S$ ) will be enhanced. This is precisely what we observe: FSRRS measurements with RP at $440 \mathrm{~nm}$ mostly reveal vibrational ground state features, whereas with RP at $460 \mathrm{~nm}$ the transitions from higher lying (less populated) vibrational levels become visible. The ability to tune the RP and exploit different resonance conditions ${ }^{31}$, jointly with the high energy resolution, allows us to clearly discriminate hot bands from anharmonic mode coupling effects. Specifically, having access to spectral features corresponding to hot bands as well as fundamental transitions, and taking into account the electronic enhancement corresponding to different RP wavelengths, the so called Raman Excitation Profile $^{32}$ (REP, see numerical model in Supplementary 

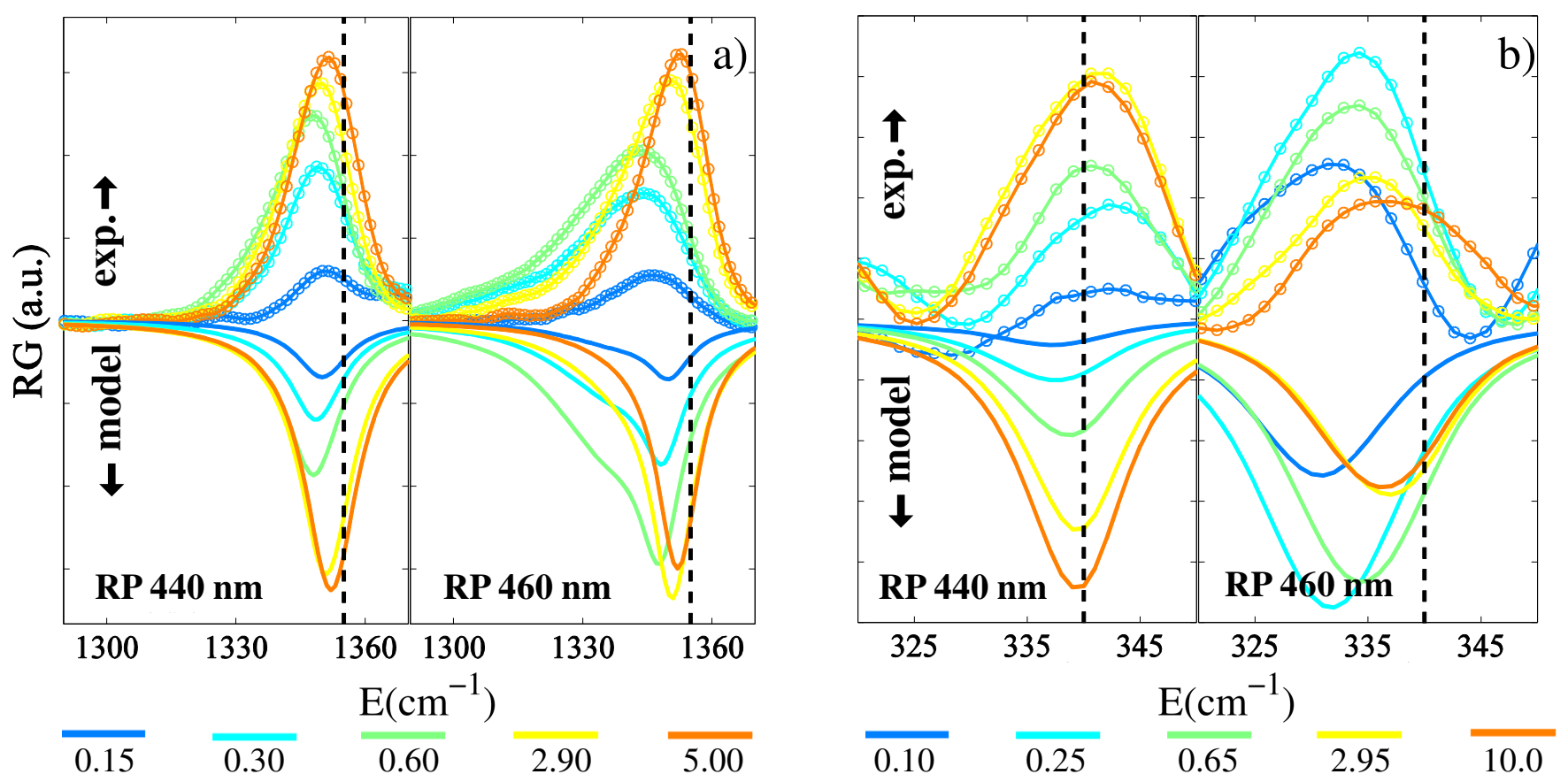

FIG. 4: Modeling sub-picosecond vibrational dynamics in Mb. Measured and modeled FSRRS experiments for $\nu_{4}$ (a) and $\nu_{8}(\mathrm{~b})$. The highest temperature reached by the two vibrational modes in the simulation are $750 \mathrm{~K}$ and $1500 \mathrm{~K}$, respectively. The corresponding anharmonicity parameters are $\alpha=0.004$ and $\alpha=0.0045$. The model accounts for TA dynamics via the evaluation of the REP profile, as detailed in Supplementary Material. The reported pump-probe delays are in picoseconds.
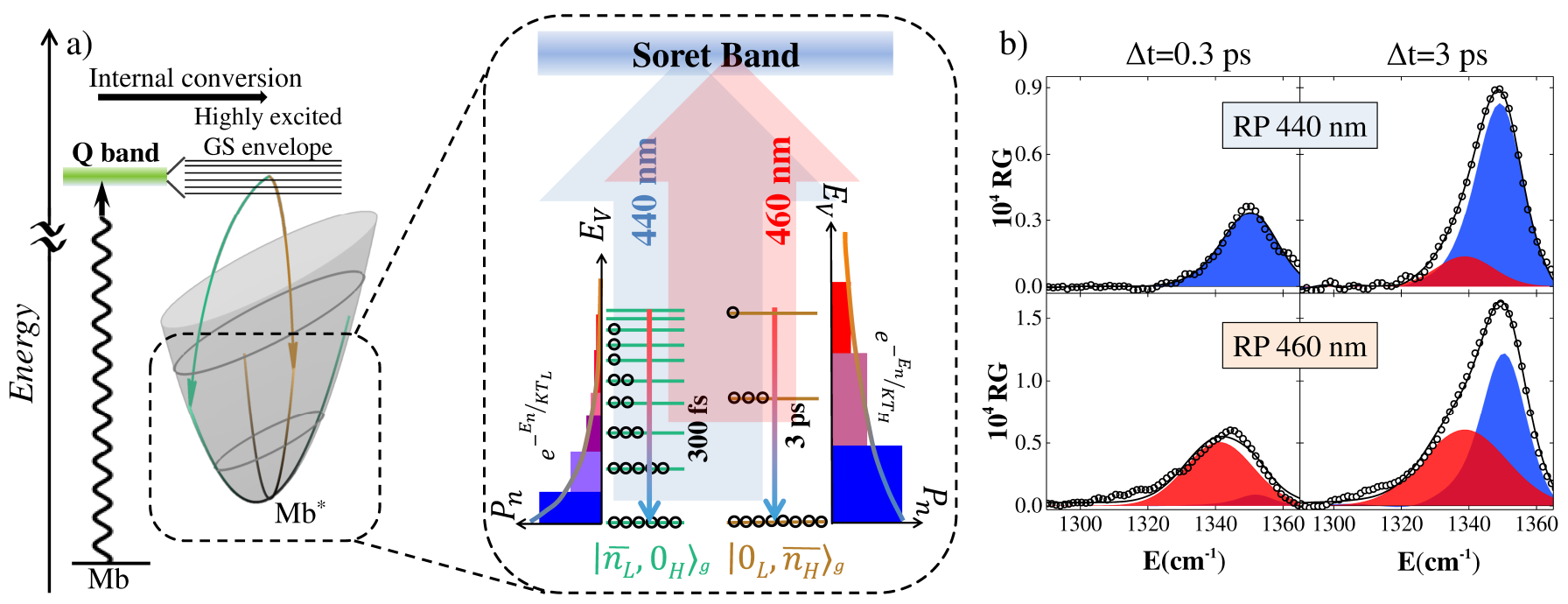

FIG. 5: Proposed energy transfer mechanism in Mb. (a) Energy transfer sketch as seen by FSRRS for different $\mathrm{RP}$ wavelength. Upon photoexcitation, energy is transfered to the highly excited vibronic manifold by FC coupling. The redistribution pathway to high and low frequency vibrational modes is then monitored by FSRRS. In the inset the behaviour of two modes representative of low $\left(\left|n_{L}\right\rangle_{g}\right)$ and high $\left(\left|n_{H}\right\rangle_{g}\right)$ frequency ground state vibrations is shown, illustrating their Boltzmann-occupation distribution $P_{n}$, represented by black circles. RP at $460 \mathrm{~nm}$, with an energy distance of $\sim 1250 \mathrm{~cm}^{-1}$ from the Soret band, emphasizes Raman transitions from vibrationally excited states. (b) experimental data (black circles) and best fits (black lines) of $\nu_{4}$ at 0.3 and 3 ps delays in deoxy-Mb. The model function is the sum of a ground state vibrational spectrum (blue area) and a Lorentzian peak representing the first hot band (red area). 
Material), we have been able to fully characterize the population of each excited vibrational level for a given Raman mode.

Within this picture, one naturally rationalizes the different evolution of high-frequency, as $\nu_{4}$ (Fig. 5b), and of low-frequency modes. In fact, on the ultrafast timescales probed here, the concept of a single equilibrium temperature no longer applies, and a hot ground state can generate different behaviors for modes of different frequency, according to the specific pathway of the intramolecular energy redistribution.

Specifically, exciting the system with a $560 \mathrm{~nm}$ photon results in a heat deposition of $53 \mathrm{kcal} / \mathrm{mole}$. This corresponds to an equilibrium average vibrational energy per mode of $\approx 500 \mathrm{~cm}^{-1}$ or, equivalently, an average vibrational temperature $700 \mathrm{~K}^{4}$. Hence, the low frequency modes such as $\nu_{8}\left(342 \mathrm{~cm}^{-1}\right)^{18}$ may have a relatively large number of vibrational levels populated by the anharmonic coupling with the initially excited FranckCondon (FC) manifold. The instantaneous redshift observed at the earliest accessible time delays in Fig. 3b signifies that those low frequency modes are the most efficient in promptly harvesting the deposited excess energy. The subsequent, gradual, blue-shift of the Raman peak indicates that the higher lying vibrational levels of the low-frequency modes quasi-continuously depopulate during the cooling process.

Under comparable heating conditions, only the two lower lying vibrational levels $\left(|0\rangle_{g}\right.$ and $\left.|1\rangle_{g}\right)$ of high frequency modes $\left(\nu>1000 \mathrm{~cm}^{-1}\right.$ such as $\left.\nu_{4}\right)$ can be significantly populated, after the system relaxes from the modes initially populated in the internal conversion process. Accordingly, only the fundamental component $0 \rightarrow 1$ and the first hot band $1 \rightarrow 2$ are observed in the FSRRS spectra, with a time dependence of the relative intensities reflecting the heating and cooling processes.

Taken together, our observations indicate the following scenario: an initial nonradiative transition from the excited state to the ground electronic state occurs through a manifold of highly excited, high frequency overtones and combination bands with the most favorable FC overlap ${ }^{33,34}$. These modes, which are extremely broad and non Raman-resonant (hence not visible in the present experiment) relax on a sub-picosecond time scale through rapid intramolecular vibrational energy redistribution. In turn, they anharmonically couple to the vibrational eigenstates of the heme, to reach a mode specific Boltzmann distribution corresponding to an elevated local vibrational temperature. Finally, slower collisional exchanges with the protein and solvent initiate the vibrational cooling process.

Critically, the evolution of the vibrational population will also be reflected in the visible absorption spectrum (see Fig. 2), as suggested in Ref. ${ }^{8}$. Specifically, we find a tight correlation between (i) the recovery timescales of the red tail of the TA $(\lambda \geq 480 \mathrm{~nm})$ and of the low frequency modes (e.g. $\left.\nu_{8}\right)(\sim 0.3$ ps timescale) ; (ii) the TA kinetics in the wavelength range $445-455 \mathrm{~nm}$ and the build up and recovery ( $\approx 3$ ps timescale) of the high frequency vibrational modes (e.g. $\left.\nu_{4}\right)$.

The first feature signifies that excess vibrational energy is very efficiently transferred from the FC envelope to the low frequency modes of the electronic ground state, increasing its average energy. Accordingly, the energy gap to the Soret level (Fig. 5a) is reduced, and the broad most redshifted component in the TA spectrum is generated. Notably, levels corresponding to the absorption of multiple low frequency quanta must necessarily be populated to produce such a relatively large shift. The second feature suggests that a similar energy transfer towards the high frequency modes is much slower, hence less efficient, and indicates an increased gap to the Soret level, reflecting dissipation of the total vibrational energy of the heme to the surrounding globin and solvent. The different timescales for the relaxation to low and high frequency modes reflect the lower vibrational density of states of the latter ${ }^{1}$.

Within this scenario, we are able to describe the observed dynamics in terms of vibrational relaxation of a hot ground state photoproduct, without invoking the presence of short-lived electronic intermediates. The possible existence of transient electronic excitations involving the d-electrons of the iron via the pi electrons of the porphyrin remains a likelihood for the non-radiative decay process ${ }^{24}$. Our result, however, sets an upper limit for their lifetime which needs to be within the FSRRS time resolution, namely $50 \mathrm{fs}$, rationalizing earlier work on the potential impact of possible photoinduced heating on $\mathrm{TA}^{8}$, resonance Raman ${ }^{2,8}$ and femtosecond impulsive vibrational coherence spectroscopy results ${ }^{35}$.

To verify this picture, we implemented a numerical model (see Supplementary Material for full details) which allows evaluation of anharmonicity factors $\left(\alpha \sim 5 \times 10^{-3}\right)$ and picosecond and sub-picosecond temperature changes for each vibrational mode $(\lesssim 1200 \mathrm{~K}$, in agreement with theoretical simulations $\left.{ }^{4}\right)$. The rationale of the model is to evaluate the Raman lineshape accounting for: (i) the temperature dependent, mode specific population dynamics of each vibrational level and (ii) the TA dynamics, affecting the spectral lineshapes via the REP (Supplementary Eq. 6). This latter feature is critical for the understanding of several key issues, as it can impact on the apparent ultrafast dynamics of the raw FSRRS spectra, making it significantly different from the population kinetics ruling energy transfer. For any vibrational transition of a given vibrational mode, indeed, the REP is in general strongly suppressed at short times, due to the bleaching and broadening of the Soret absorption band (see Fig. 2). Consequently, FSRRS dynamics at short times are only visible in those cases where population dynamics compensate for the small REP. As an example, although the vibrational ground state of the high 
frequency modes (see $\nu_{4}$ in Supplementary Fig. 3) remains populated above $90 \%$ during the entire dynamics, the spectrum corresponding to the fundamental vibronic transition is obscured during the first picosecond. For low frequency modes, on the other hand, the spectra show similar behavior only for the ground state resonant measurement at $440 \mathrm{~nm}$ (where both the REP and $n=0$ population monotonically increase with time). The 460 $\mathrm{nm}$ resonance, indeed, emphasizes the interplay between the rise of the REP and the decay of the vibrationally excited levels (see $\nu_{8}$ in Supplementary Fig. 3).

As shown in Fig. 4, the model quantitatively reproduces the experiment, showing that the energy transfer to the low frequency modes is more efficient than that to high frequency vibrations.

Vibrational heating on the picoseconds timescale has been studied in the past by looking at the Stokes/antiStokes ratio in $\mathrm{TR}^{3}$ and $\mathrm{TRRS}^{2,16}$. Very recently, however, it has been pointed out that the Stokes/anti-Stokes ratio of stimulated Raman spectra is insensitive to the local temperature ${ }^{36}$. Tracking hot bands dynamics in a FSRRS experiment, the method proposed in this paper, represents therefore a powerful tool to capitalize on the sub-ps capabilities of the technique. It allows, indeed, mapping the earliest stages of energy flow at the intramolecular scale, namely at the ligand binding site of a heme protein, by tracking local temperature dynamics. In line with previous TRRS results ${ }^{37}$ (see Supplementary Discussion for an extended examination), we identify a bottleneck role of high frequency modes ${ }^{2,22}$ on the way to thermal equilibrium, which can be explained as trapping of vibrational energy in those modes located within a low density of states region ${ }^{38}$.

Finally, the tight correlation between the kinetics of the TA and the vibrational evolution permits describing energy flow uniquely in terms of a hot ground state relaxation, ruling out a role of transient electronic states ${ }^{21}$ on the timescale $>50$ fs. By studying heme proteins with a variety of functions, including proteins incorporating covalently linked heme like cytochrome c, our approach offers the perspective of mapping out in detail the modes involved in energy exchange between a cofactor and the protein backbone. These include the modes orchestrating the initial stages of intra-protein signalling processes upon heme-ligand bond-breaking, with implications for functional roles such as protein cooling during reaction, allosteric communication and efficient ligand binding and trapping $^{27}$. On a more fundamental ground, our results challenge the common understanding of temperature as an equilibrium molecular property, elucidating its modespecific character.

\section{Methods}

FSRRS method - A possible route to supersede the picosecond time resolution limit, without the loss of spectral resolution typical of spontaneous Raman, is stimulating Raman transition by the simultaneous presence of two electric fields (RP and Stokes probe) ${ }^{39,40}$. Using dispersed detection, indeed, spectral resolution is fundamentally limited by the RP bandwidth. Nonetheless, the time resolution is only determined by the duration of the Stokes probe pulse initiating the macroscopic polarization in the sample and, of course, by the actinic pump. Starting from the seminal paper on retinal isomerisation ${ }^{41}$, this experimental protocol has been demonstrated in a number of molecular systems (see ${ }^{42}$ for a comprehensive review) and recently extended to condensed matter to tackle ultrafast light-induced modifications of the magnetic interaction ${ }^{43}$.

In the last few years, we introduced an efficient technique to synthesise broadly tunable narrowband picosecond pulses ${ }^{44,45}$, which we use here as RP for an FSRRS experiment selectively exploring different Soret resonance conditions. The optical layout of the experiment is detailed in Ref. ${ }^{44}$. Briefly, the setup is driven by an amplified Ti:Sapphire laser (Coherent Legend Elite $\mathrm{HE})$ producing $3.6-\mathrm{mJ}, 35$-fs pulses at $800 \mathrm{~nm}\left(450 \mathrm{~cm}^{-1}\right.$ bandwidth) and $1 \mathrm{kHz}$ repetition rate. Widely tunable RPs are an essential component of the setup. The narrow bandwidth RP in the range $350-600 \mathrm{~nm}$ is generated from a two-stage Optical Parametric Amplifier (OPA) that produces tunable IR-visible pulses, followed by spectral compression via frequency doubling in a 25$\mathrm{mm}$ beta-barium borate (BBO) crystal. Spectral compression technique takes advantage of the group velocity mismatch between the fundamental and second harmonic in the long BBO crystal to create few-ps duration pulses with typical bandwidths of $\sim 15 \mathrm{~cm}^{-1}$. The temporal profile of the RP is then shaped via spectral filtering, as we recently detailed in Ref. ${ }^{45}$. The femtosecond Stokes probe is a white-light continuum generated by focusing the laser fundamental into a $\mathrm{CaF}_{2}$ crystal that is continuously translated perpendicular to the beam to prevent damage. The actinic pump, which triggers the photoexcitation, is generated by a second OPA tuned to $\lambda=560 \mathrm{~nm}$, i.e. in resonance with the Q-band absorption of $\mathrm{Mb}$. The energies of the beams are $\sim 1 \mu \mathrm{J}, \sim 0.7 \mu \mathrm{J}$ and $\sim 2 \mathrm{~nJ}$ for the actinic pump, RP and Stokes pulse, respectively. The three beams are independently focused onto the samples, the RP is modulated at $500 \mathrm{~Hz}$ to obtain stimulated Raman gain (RG), while the actinic pump is modulated at $250 \mathrm{~Hz}$ to obtain the FSRRS difference spectra upon photoexcitation.

Sample preparation - Horse's heart Myoglobin was purchased from Sigma Aldrich, dissolved in 0.1 M phosphate buffer, $\mathrm{pH} 7.5$, and reduced under anaerobic con- 
ditions with sodium dithionite to the deoxy ferrous state. The CO complex was formed by exposing this state to $\mathrm{CO}$ gas. Typical sample concentrations are in the range 100 and $200 \mu \mathrm{M}$. To avoid radiation damage, the sample flows through a transmission cell, by means of a peristaltic pump.

Data treatment - To correctly calibrate the Raman spectrum, cyclohexane was preliminarly used as a standard. To obtain the Mb FSRRS spectrum two unwanted contributions need to be removed from the data: a TA induced background, and the stimulated Raman contribution of the not-reactive part of sample. The former contribution arises in resonance condition, as the white light continuum is absorbed differently in presence and in absence of the RP (see Supplementary Fig. 1). In other words $I_{\text {Ron }}$ and $I_{\text {Roff } f}$ in Supplementary Fig. 1 do not differ by the Raman spectrum only ${ }^{46}$. The most simple correction is possible by fitting the baseline with a spline $^{47}$ and dividing it from the spectrum ${ }^{4-50}$. In our case the spline is applied with variable values of interpolant, and limited to wavelength regions not involved in the FSRRS effects (see Supplementary Fig. 1). To remove the Raman spectrum related to non-photoexcited molecules, we subtracted an increasing amount of signal without the actinic pump (AP-off), up to the onset of negative contributions in the Raman gain, similarly to $\operatorname{Ref}^{51}$.

A further important reduction of the experimental data (Fig. 3) concerns the equalization of pump-probe time delays, which are different for each vibrational feature, owing to the chirp of the broadband Stokes pulse. To this purpose, we used Optical Kerr Effect (OKE) cross-correlation $^{40}$ to measure the overlap between the actinic pump and the broadband Stokes pulse.

1. Leitner, D. M. Energy flow in proteins. Annu. Rev. Phys. Chem. 59, 233-259 (2008).

2. Mizutani, Y. \& Kitagawa, T. Direct observation of cooling of heme upon photodissociation of carbonmonoxy myoglobin. Science 278, 443-446 (1997).

3. Miller, R. J. D. Vibrational energy relaxation and structural dynamics of heme proteins. Annu. Rev. Phys. Chem. 42, 581-614 (1991).

4. Henry, E. R., Eaton, W. A. \& Hochstrasser, R. M. Molecular dynamics simulations of cooling in laser-excited heme proteins. Proc. Natl. Acad. Sci. U.S.A. 83, 8982-8986 (1986).

5. Mukamel, S. Principles of Nonlinear optical spectroscopy (Oxford University Press, 1995).

6. Brunori, M. Myoglobin strikes back. Protein Sci. 19, 195-201 (2010).

7. Lim, M., Jackson, T. A. \& Anfinrud, P. A. Femtosecond near-IR absorbance study of photoexcited myoglobin: Dynamics of electronic and thermal relaxation. J. Phys. Chem. 100, 12043-12051 (1996).

8. Ye, X. et al. Investigations of heme protein absorption line shapes, vibrational relaxation, and resonance raman scattering on ultrafast time scales. J. Phys. Chem. A 107, 8156-8165 (2003).

9. Ye, X., Demidov, A. \& Champion, P. M. Measurements of the photodissociation quantum yields of mbno and mbo2 and the vibrational relaxation of the six-coordinate heme species. J. Am. Chem. Soc. 124, 5914-5924 (2002).

10. Kholodenko, Y., Volk, M., Gooding, E. \& Hochstrasser, R. Energy dissipation and relaxation processes in deoxy myoglobin after photoexcitation in the soret region. Chem. Phys. 259, 71-87 (2000).

11. Armstrong, M. R., Ogilvie, J. P., Cowan, M. L., Nagy, A. M. \& Miller, R. J. D. Observation of the cascaded atomic-to-global length scales driving protein motion. Proc. Natl. Acad. Sci. U.S.A. 100, 4990-4994 (2003).

12. Groot, M.-L. et al. Coherent infrared emission from myoglobin crystals: An electric field measurement. Proc. Natl. Acad. Sci. U.S.A. 99, 1323-1328 (2002).

13. Franzen, S., Bohn, B., Poyart, C. \& Martin, J. L. Evidence for sub-picosecond heme doming in hemoglobin and myoglobin: a time-resolved resonance raman comparison of carbonmonoxy and deoxy species. Biochemistry 34, 1224-1237 (1995).

14. Petrich, J. W., Martin, J. L., Houde, D., Poyart, C. \& Orszag, A. Time-resolved raman spectroscopy with subpicosecond resolution: vibrational cooling and delocalization of strain energy in photodissociated (carbonmonoxy)hemoglobin. Biochemistry 26, 7914-7923 (1987).

15. Kruglik, S. G., Lambry, J.-C., Martin, J.-L., Vos, M. H. \& Negrerie, M. Sub-picosecond raman spectrometer for time-resolved studies of structural dynamics in heme proteins. J. Raman Spectrosc. 42, 265-275 (2011).

16. Simpson, M. C. et al. Transient raman observations of heme electronic and vibrational photodynamics in deoxyhemoglobin. J. Am. Chem. Soc. 119, 5110-5117 (1997).

17. Li, P., Sage, J. T. \& Champion, P. M. Probing picosecond processes with nanosecond lasers: Electronic and vibrational relaxation dynamics of heme proteins. J. Chem. Phys. 97, 3214-3227 (1992).

18. Li, X. Y., Czernuszewicz, R. S., Kincaid, J. R., Stein, P. \& Spiro, T. G. Consistent porphyrin force field. 2. nickel octaethylporphyrin skeletal and substituent mode assignments from nitrogen-15, meso-d4, and methylened16 raman and infrared isotope shifts. J. Phys. Chem. 94, 47-61 (1990).

19. Cornelius, P. A., Steele, A. W., Chernoff, D. A. \& Hochstrasser, R. M. Different dissociation pathways and observation of an excited deoxy state in picosecond photolysis of oxy- and carboxymyoglobin. Proc. Natl. Acad. Sci. U.S.A. 78, 7526-7529 (1981).

20. Lim, M., Jackson, T. \& Anfinrud, P. Binding of CO to myoglobin from a heme pocket docking site to form nearly linear Fe-C-O. Science 269, 962-966 (1995).

21. Petrich, J. W., Poyart, C. \& Martin, J. L. Photophysics and reactivity of heme proteins: a femtosecond absorption study of hemoglobin, myoglobin, and protoheme. Biochemistry 27, 4049-4060 (1988).

22. Schneebeck, M., Vigil, L. \& Ondrias, M. Mode-selective energy localization during photoexcitation of deoxyhemoglobin and heme model complexes. Chem. Phys. Lett. 215, 251-256 (1993).

23. Franzen, S., Kiger, L., Poyart, C. \& Martin, J.-L. Heme photolysis occurs by ultrafast excited state metal-to-ring charge transfer. Biophys. J. 80, 2372-2385 (2001). 
24. Consani, C., Auböck, G., Bräm, O., van Mourik, F. \& Chergui, M. A cascade through spin states in the ultrafast haem relaxation of met-myoglobin. J. Chem. Phys. 140, 025103 (2014).

25. Levantino, M. et al. Ultrafast myoglobin structural dynamics observed with an X-ray free-electron laser. Nat. Commun. 6 (2015).

26. Kukura, P., McCamant, D. W. \& Mathies, R. A. Femtosecond stimulated raman spectroscopy. Annu. Rev. Phys. Chem. 58, 461-488 (2007).

27. Champion, P. M. Following the flow of energy in biomolecules. Science 310, 980-982 (2005).

28. Mukamel, S. \& Biggs, J. D. Communication: Comment on the effective temporal and spectral resolution of impulsive stimulated raman signals. J. Chem. Phys. 134, 161101 (2011).

29. Fumero, G., Batignani, G., Dorfman, K. E., Mukamel, S. \& Scopigno, T. On the Resolution Limit of Femtosecond Stimulated Raman Spectroscopy: Modelling Fifth-Order Signals with Overlapping Pulses. ChemPhysChem 16, 3438-3443 (2015).

30. Sagnella, D. E., Straub, J. E., Jackson, T. A., Lim, M. \& Anfinrud, P. A. Vibrational population relaxation of carbon monoxide in the heme pocket of photolyzed carbonmonoxy myoglobin: Comparison of time-resolved mid-IR absorbance experiments and molecular dynamics simulations. Proc. Natl. Acad. Sci. U.S.A. 96, 14324-14329 (1999).

31. Batignani, G. et al. Electronic resonances in broadband stimulated Raman spectroscopy. Scientific Reports 6, 18445 (2016).

32. Stallard, B. R., Champion, P. M., Callis, P. R. \& Albrecht, A. C. Advances in calculating raman excitation profiles by means of the transform theory. J. Chem. Phys. 78, 712-722 (1983).

33. Siebrand, W. Radiationless transitions in polyatomic molecules. ii. tripletgroundstate transitions in aromatic hydrocarbons. The Journal of Chemical Physics 47, 2411-2422 (1967).

34. Englman, R. \& Jortner, J. The energy gap law for radiationless transitions in large molecules. Molecular Physics 18, 145-164 (1970).

35. Rosca, F. et al. Investigations of anharmonic lowfrequency oscillations in heme proteins. J. Phys. Chem. A 106, 3540-3552 (2002).

36. Harbola, U., Umapathy, S. \& Mukamel, S. Loss and gain signals in broadband stimulated-raman spectra: Theoretical analysis. Phys. Rev. A 88, 011801 (2013).

37. Loparo, J. J., Cheatum, C. M., Ondrias, M. R. \& Simpson, M. Transient raman observations of heme vibrational dynamics in five-coordinate iron porphyrins. Chem. Phys. 286, 353-374 (2003).

38. Leitner, D. M. Frequency-resolved communication maps for proteins and other nanoscale materials. J. Chem. Phys. 130, 195101 (2009).

39. Yoshizawa, M., Hattori, Y. \& Kobayashi, T. Femtosecond time-resolved resonance raman gain spectroscopy in polydiacetylene. Phys. Rev. B 49, 13259-13262 (1994).

40. McCamant, D. W., Kukura, P., Yoon, S. \& Mathies, R. A. Femtosecond broadband stimulated raman spectroscopy: Apparatus and methods. Rev. Sci. Instrum. 75, 49714980 (2004).

41. Kukura, P., McCamant, D. W., Yoon, S., Wandschneider, D. B. \& Mathies, R. A. Structural observation of the pri- mary isomerization in vision with femtosecond-stimulated raman. Science 310, 1006-1009 (2005).

42. Dietze, D. R. \& Mathies, R. A. Femtosecond Stimulated Raman Spectroscopy. ChemPhysChem 17, 12241251 (2016).

43. Batignani, G. et al. Probing ultrafast photo-induced dynamics of the exchange energy in a Heisenberg antiferromagnet. Nat Photon 9, 506-510 (2015).

44. Pontecorvo, E. et al. Femtosecond stimulated raman spectrometer in the 320-520nm range. Opt. Express 19, 11071112 (2011)

45. Pontecorvo, E., Ferrante, C., Elles, C. G. \& Scopigno, T. Spectrally tailored narrowband pulses for femtosecond stimulated raman spectroscopy in the range $330-750 \mathrm{~nm}$. Opt. Express 21, 6866-6872 (2013).

46. Weigel, A. \& Ernsting, N. Excited stilbene: Intramolecular vibrational redistribution and solvation studied by femtosecond stimulated raman spectroscopy. J. Phys. Chem. B 114, 7879-7893 (2010).

47. Pontecorvo, E., Ferrante, C., Elles, C. G. \& Scopigno, T. Structural rearrangement accompanying the ultrafast electrocyclization reaction of a photochromic molecular switch. J. Phys. Chem. B 118, 6915-6921 (2014).

48. Laimgruber, S., Schachenmayr, H., Schmidt, B., Zinth, W. \& Gilch, P. A femtosecond stimulated raman spectrograph for the near ultraviolet. Appl. Phys. B 85, 557-564 (2006).

49. Kloz, M., van Grondelle, R. \& Kennis, J. T. Correction for the time dependent inner filter effect caused by transient absorption in femtosecond stimulated raman experiment. Chem. Phys. Lett. 544, $94-101$ (2012).

50. Weigel, A. et al. Femtosecond stimulated raman spectroscopy of flavin after optical excitation. J. Phys. Chem. $B$ 115, 3656-3680 (2011).

51. Kruglik, S. G. et al. Picosecond primary structural transition of the heme is retarded after nitric oxide binding to heme proteins. Proc. Natl. Acad. Sci. U.S.A. 107, 1367813683 (2010).

Acknowledgements TS thanks A. Arcovito for early discussions on the potential impact of an FSRS experiment in myoglobin, and gratefully acknowledges an inspiring visit at the Mathies lab.

M. Aschi, G. Batignani, P. Champion, M. Garavelli, P. Kukura, Y. Mizutani, S. Kruglik and S. Mukamel provided invaluable input to this work.

The authors thank B. Vallone and the Dipartimento di Scienze Biochimiche and Istituto Pasteur-Fondazione Cenci Bolognetti of Università di Roma "La Sapienza" for the support with sample preparation.

TS is specially grateful to M. Brunori for continued support and critical insights.

This research has received funding from the European Research Council under the European Unions Seventh Framework Program (FP7/2007-2013) and No. 207916 (FEMTOSCOPY).

Author Contributions T.S. directed the research. E.P. led the experimental activity with the assistance of C.F. and support from G.C. and T.S.; C.F. performed data analysis and numerical modeling with the assistance of E.P. T.S. wrote the manuscript with C.F. and M.V. 
All authors discussed the results and implications and

commented on the manuscript. 